\title{
ANÁLISIS PRAGMÁTICO DE DOS EXPRESIONES DE CORTESÍA DEL ESPAÑOL DE COSTA RICA Y SU ADQUISICIÓN POR PARTE DE ESTUDIANTES DE ESPAÑOL COMO SEGUNDA LENGUA
}

\author{
Soledad Urbina Vargas
}

\begin{abstract}
RESUMEN
Este artículo propone el análisis, en el campo de la adquisición pragmática de una segunda lengua y específicamente dentro del desarrollo de estrategias de cortesía e identificación de actos de habla, de dos expresiones: "un día de estos hagamos algo" y "un día de estos llegáte a mi casa". La investigación contrasta la percepción y la identificación de las frases en estudio como un acto de habla específico, en hablantes nativos y aprendices de español como segunda lengua.

Palabras clave: Pragmática, cortesía, segundas lenguas, español.
\end{abstract}

\section{ABSTRACT}

This article proposes the analysis, within the field of the pragmatic acquisition of a second language and specifically within the field of the development of strategies of politeness strategies and identification of speech acts, of two phrases "un día de estos llegáte a mi casa" and "un día de estos hagamos algo". The investigation will show contrast between the perception and the identification of these phrases under study as a specific speech act, in native speakers and Spanish L2 learners.

Key words: Pragmatics, politeness, second languages, spanish.

\section{Las estrategias de cortesía}

En el campo de la enseñanza-aprendizaje de la pragmática de una segunda lengua, es imprescindible dotar a los estudiantes, como parte de su competencia en la lengua meta, de estrategias conversacionales de cortesía que resulten en un cumplimiento eficaz de los objetivos comunicativos.

Como apunta Escandel Vidal (1996: 139-41), la cortesía es una estrategia al servicio de las relaciones sociales (intercambio interaccional), opuesta al intercambio en el cual la

Soledad Urbina Vargas. Profesora de la Escuela de Filología, Lingüística y Literatura de la Universidad de Costa Rica. San Pedro, San José, Costa Rica. 
transmisión eficaz de la información es prioritaria, es decir, cuando existe una necesidad particular de transmitir concisamente el mensaje (intercambio transaccional o proposicional).

Mediante la cortesía, los hablantes marcan las relaciones sociales: la familiaridad, el respeto, la atenuación de acciones menos corteses. Al adquirir una lengua se debe contar con este tipo de conocimiento con el fin de poder interactuar dentro de una comunidad.

Leech (1983: 83), en relación con el uso de la cortesía señala:

\footnotetext{
Algunas ilocuciones (por ejemplo, las órdenes) son inherentemente descorteses; y otras (por ejemplo, los ofrecimientos) son inherentemente corteses.
}

Para Leech, la acción es más descortés entre mayor es el coste para el destinatario y menor su beneficio; y es más cortés cuando el coste es mayor para el emisor y el beneficio mayor para el destinatario.

Con base en la clasificación general de diferentes actos de habla de Leech y en relación con lo postulado por el investigador en cuanto a la escala de mayor o menor cortesía de las acciones y su impacto sobre las relaciones sociales, se establece la existencia de:

I) Acciones que apoyan la cortesía, es decir, que mantienen o mejoran las relaciones sociales existentes entre ellos: son acciones como agradecer, felicitar, saludar, invitar...

II) Acciones indiferentes a la cortesía, en las que no hay un desequilibrio claro entre coste y beneficio para el emisor y el destinatario: afirmar, informar, anunciar...

III) Acciones que entran en conflicto con la cortesía, es decir, el coste del destinatario es mayor: pedir, ordenar...

IV) Acciones en contra de la relación entre los interlocutores, las cuales pretenden aumentar la distancia social o destruir las relaciones existentes: amenazar, acusar, maldecir...

Se aprecia que dentro de los actos (acciones) de habla corteses, se encuentra la acción de saludar, descrita por Haverkate (1994: 84) como un acto expresivo idiosincrásico, quien apunta las siguientes razones:

\footnotetext{
El saludo se considera como un acto expresivo universal, ya que es un componente verbal integrado en la interacción social de todas las culturas.

Su realización formal está basada en fórmulas rutinarias, generalmente fosilizadas.

El acto de saludar no sirve para transmitir información proposicional.
}

Haverkate señala que los saludos no se intercambian, a diferencia de otros actos de habla, para expresar una descripción del mundo extralingüístico; por esa razón, en su realización intervienen más signos paralingüísticos y no lingüísticos.

Entre las más importantes funciones intercomunicativas del saludo, dependiendo de cual sea nuestra selección a la hora de estructurarlo, se establecen diferentes relaciones entre los interlocutores, por ejemplo, posición social, grado de confianza o grado de afecto.

Los enunciados en estudio "un día de estos llegáte a mi casa” (enunciado A) y "un día de estos hagamos algo" (enunciado B) son fórmulas de saludo informales empleadas en un acto de habla cortés como es el saludar; es decir, su uso es meramente convencional y, según opiniones de los hablantes nativos (ver apéndice 2), se utilizan cuando hay confianza con el interlocutor, como una forma de "hacerlo (la) sentir bien". 
En distintas ocasiones, los profesores de segunda lengua reciben quejas de los estudiantes en cuanto a la falta de cortesía de los ticos a la hora de cumplir con las invitaciones hechas; contribuye grandemente a esta confusión la falta de conocimiento de estrategias de cortesía por parte de los estudiantes, quienes interpretan literalmente frases como las que se analizan.

La acción de saludar presenta dos momentos: la apertura de la interacción y el cierre de la comunicación verbal (saludo-despedida). Partimos de la percepción de que ambas expresiones estudiadas pertenecen a la categoría de cierre de la comunicación verbal. No obstante, en la investigación se pone a prueba la identificación y la percepción de las expresiones (A y B) como fórmulas de despedida informales, tanto para los hablantes no nativos como para los nativos.

\section{Recolección de los datos}

Con el objetivo de analizar la identificación e interpretación de los enunciados (A y B) por parte de los hablantes no nativos, se diseñó un cuestionario (ver apéndice 1), el cual se dividió en dos secciones. La primera era de escogencia múltiple (multiple-choice); en la otra sección, el hablante debía completar la conversación (Discurse Completion tasks). El cuestionario también se aplicó a hablantes nativos del español (20 en total) con el fin de contrastar los resultados y verificar el empleo de las fórmulas como posibles cierres de un saludo.

La muestra de hablantes no nativos de español ${ }^{1}$ (20 en total) fue restringida a aquellos con estudios formales en el español como segunda lengua, estudiantes de niveles avanzados (intermedio alto, avanzado) cuya permanencia en el país fuera de tres meses en adelante, con el propósito de que su competencia, tanto por el nivel de exposición como por el tiempo en que habían estado estudiando formalmente, fuera mayor.

Ya que el interés primordial era el reconocimiento y la percepción del acto de habla, se empleó una situación (diálogo) en la cual se estableció una relación informal (entre amigos) y sin jerarquías entre los interlocutores.

\section{Resultados}

Mientras que los hablantes no nativos se orientan hacia el elemento proposicional, es decir, hacia el sentido literal de la estructura gramatical, los nativos tienden a interpretar los enunciados como parte de una situación (ver apartado b del apéndice 1).

Diferencias encontradas al comparar las respuestas de ambos grupos:

\subsection{Identificación del enunciado "un día de estos llegáte a mi casa" (E.A)}

La mayoría de los hablantes no nativos lo identifican como una invitación. 


\section{Cuadros 1 y 2}

\begin{tabular}{|l|l|}
\hline \multicolumn{2}{|l|}{ Respuesta de los hablantes no nativos } \\
\hline Invitación & 9 \\
\hline Invitación y despedida & 4 \\
\hline Invitación y saludo & 1 \\
\hline Mandato & 6 \\
\hline
\end{tabular}

\begin{tabular}{|l|l|}
\hline \multicolumn{2}{|l|}{ Respuesta de los hablantes nativos } \\
\hline Invitación & 6 \\
\hline Invitación y despedida & 10 \\
\hline Invitación y saludo & 4 \\
\hline Mandato & 0 \\
\hline
\end{tabular}

\subsection{Identificación del enunciado "un día de estos hagamos algo" (EB)}

La mayoría de los hablantes no nativos identifican el enunciado "un día de estos hagamos algo" como una invitación.

Cuadros 3 y 4

\begin{tabular}{|l|l|}
\hline \multicolumn{2}{|l|}{ Respuesta de los hablantes no nativos } \\
\hline Invitación & 15 \\
\hline Invitación y despedida & 4 \\
\hline Saludo & 1 \\
\hline Mandato & 0 \\
\hline
\end{tabular}

\begin{tabular}{|l|l|}
\hline \multicolumn{2}{|l|}{ Respuesta de los hablantes nativos } \\
\hline Invitación & 4 \\
\hline Invitación y despedida & 11 \\
\hline Saludo & 4 \\
\hline Mandato & 1 \\
\hline
\end{tabular}

\subsection{Resultados al completar el diálogo}

En el ítem donde se pedía completar el diálogo, la mayoría de los hablantes no nativos utilizaron enunciados que no eran correctos - desde un punto de vista pragmático- para la situación; es decir, fórmulas no correspondientes a las empleadas por los hablantes nativos.

3.3.1. Respuestas de los extranjeros para la fórmula "un día de estos llegáte a mi casa" (E.A)

a. “¡Ojalá que el próximo fin de semana!”

b. "Sí, me gustaría hacerlo"

c. "Está bien, voy a venir (ir)"

d. "Sí, mañana te visitaré"

e. "Voy a llegar el martes"

f. "Sí, la semana próxima"

g. "¿Claro!, no trabajo mañana, podemos almorzar" 
3.3.2. Respuestas de los hablantes nativos para la fórmula"un día de estos llegáte a mi casa"
a. "¡Bueno, un día de estos me llego!"
b. "¡Por supuesto!"
c. "¡Claro!, ipero que no se vuelva paja!"
d. "iLlamo para ponernos de acuerdo!"
e. "Me parece, ¡nos estamos hablando!"
f. "¡Huy!, sí ¡tuanis! (anglicismo por too nice) ¡Ahí te llamo!”
g. "De acuerdo, llego un día de estos (a la tica)"

3.3.3. Respuestas de los extranjeros para la fórmula "un día de estos hagamos algo" (E.B)
a. "Sí ¿Qué quieres hacer?"
b. "OK. Vamos al cine"
c. "Sí, me gustaría ¿cuándo?"
d. "¿Cómo qué? Yo quiero ir al teatro"
e. "Quería hacer algo con usted"
f. "Bueno, te llamaré en cuanto tenga tiempo"
g. "Sí, está bien"
h. "Sí, te llamo para ponernos de acuerdo"

\subsubsection{Respuestas de los hablantes nativos para "un día de estos hagamos algo"}
a. "¡Por supuesto!"
b. "Te llamo!"
c. "iClaro!, ipero que no se vuelva paja!"
d. "iLlamo para ponernos de acuerdo!"
e. "Me parece, ¡nos hablamos!"
f. " ¡Cool! Yo te llamo"

Las respuestas muestran que la mayoría de los hablantes nativos identificaron los enunciados A y B, a la hora de escoger el acto de habla en el ítem de escogencia múltiple, como una invitación y una despedida, resultado que parece tener una relación directa con la forma lingǘstica, ya que cuando deben completar el diálogo y dar una respuesta a ambos enunciados, ningún nativo concreta la invitación de forma directa; el enunciado es percibido como una fórmula de despedida, o como algunos señalan tanto dentro del cuestionario como en el juicio valorativo (apéndice 2), "una invitación a la tica", comentario que sugiere el uso de una fórmula convencional, cuyo significado va más allá de la información lingüística.

Por otra parte, en los hablantes de español como segunda lengua existe una fuerte tendencia a completar el diálogo concretando, de alguna manera, la invitación.

Es importante señalar como dato interesante que hubo dos respuestas al enunciado B "sí, está bien" y "te llamo para ponernos de acuerdo", las cuales corresponden con las expresadas por los hablantes nativos y fueron formuladas por los dos estudiantes de origen latino (italianos). No fue posible someterlas a ningún análisis de transferencia pragmática ya que la información en el corpus es muy limitada y los objetivos del trabajo son de otra naturaleza. 
Además, para corroborar el significado de los enunciados dentro del contexto, se les preguntó directamente a los hablantes nativos, después de la aplicación del cuestionario, qué significado tenían las expresiones "un día de estos llegáte a mi casa" y "un día de estos hacemos algo"en la situación en que fueron presentadas, a lo cual contestaron que no las percibían como una invitación directa, que era una señal de confianza entre gente amiga y que, en general, nunca presionarían o pondrían en compromiso a su interlocutor señalando una fecha o actividad específica como una posible respuesta a las expresiones en estudio. Por tanto, es percibida como una fórmula convencional de despedida por parte de los hablantes nativos del español de Costa Rica.

\section{Conclusiones}

Cuando los aprendices de una segunda lengua se enfrentan con una nueva cultura, es necesario que cuenten tanto con conocimientos gramaticales de la lengua meta como con un conocimiento pragmático que les permita usar en contextos específicos las formas lingüísticas aprendidas, sobre todo cuando el uso y la interpretación de lo comunicado tiene relación directa con elementos culturales y sociales.

Este trabajo muestra la falta de manejo estratégico por parte del grupo de hablantes de español como segunda lengua, de los enunciados (A y B) como expresiones corteses de despedida del español de Costa Rica. Las respuestas a las expresiones en estudio por parte de los hablantes no nativos distan mucho de las formuladas por los hablantes nativos, hecho que probablemente resultaría en malos entendidos en el caso de una situación comunicativa en la que se emplearan los enunciados.

Con el propósito de enfatizar en la importancia del componente pragmático en la enseñanza del español como segunda lengua, es importante recordar que el error gramatical generalmente es "perdonado" como una falta de conocimiento de formas lingüísticas; no obstante, el fallo en el nivel pragmático provoca un malentendido personal, es decir, puede resultar en criterios negativos y errados sobre nuestro interlocutor, tales como "es una persona tan maleducada", "no es cortés", "es demasiado directa (o)".

En el proceso de enseñanza-aprendizaje de la adquisición de una segunda lengua, deben incluirse las estrategias de cortesía que se emplean en la sociedad en la que están inmersos los aprendices, con el fin de que desarrollen paralelamente una competencia lingüística y pragmática que les permita una actuación eficaz en situaciones comunicativas específicas.

Este trabajo analiza una situación comunicativa concreta; no obstante, busca dejar patente la necesidad de incluir conocimientos pragmáticos, de una forma gradual y sistemática, en cada uno de los niveles de adquisición de una segunda lengua, como herramienta eficaz en la integración del hablante de segunda lengua a la nueva cultura.

\section{Notas}

1. La muestra se obtuvo gracias a la participación de 20 estudiantes extranjeros de español como segunda lengua, quienes cursaban los niveles Intermedio II y Avanzado I en dos instituciones de educación superior costarricenses: la Universidad de Costa Rica y la Universidad Veritas. También se recibió la ayuda de 25 hablantes nativos a quienes se les aplicó el cuestionario. 


\section{Bibliografía}

Cortés Rodríguez, Luis. 2002. "La incorporación de los estudios pragmáticos al análisis del discurso oral en español”. Tonos Digital (revista electrónica de estudios filológicos). 3 (3). Disponible en http://www.tonosdigitales.com.

Bou Franch, Patricia. 1998. “On pragmatic transfer”. Studies in English Languages and Linguistics. 0: 5-20.

Bravo, Diana (ed.). 2003. Primer coloquio del Programa EDICE. Estocolmo: Universidad de Estocolmo.

Clahsen, Harald y William Rutheford. 1984. Language acquisition and language disorders. Amsterdam/Philadelphia: John Benjamins Publishing Company.

Escandel Vidal, Victoria.1996. Introducción a la Pragmática. Barcelona: Editorial Ariel.

Haverkate, Henk. 1994. La cortesía verbal. Madrid: Editorial Gredos.

Kasper, Gabriele y Kenneth R. Rose. 2002. "Pragmatic development in a second languages". Language Learning. 52 (1): 63-116.

Larsen-Freeman, Diane y Michael H. Long. 1994. Introducción al estudio de la adquisición de segundas lenguas. Madrid: Gredos.

Leech, G.N. 1983. Principles of pragmatics. London: Longman.

Piatti, Guillermina. 2003. "La cortesía: un contenido funcional para los programas de español como lengua extranjera”. En: Bravo (ed.), 355-397.

White, Lydia. 1989. “Universal grammar and second language acquisition”. En: Clahsen y Rutheford (eds.), 181-184 


\section{Anexo 1}

\section{Cuestionario para identificación y percepción de actos de habla}

Por favor, si usted no es hablante nativo de español, bríndenos la siguiente información:

1. Nivel de español:

$\square$ Principiante $\square$ Intermedio $\square$ Avanzado

2. ¿Durante cuánto tiempo ha estudiado español?

$\square$ seis meses $\square$ un año $\square$ cuatro años $\square$ más de cuatro años

3. ¿Hace cuánto tiempo vive en Costa Rica?

Respuesta:

1. Primera situación: Ana se encuentra con su amigo Mario a quien tiene algún tiempo de no ver y dice:

Ana - ¡Diay!, Mario, tanto tiempo sin verte ¿Cómo estás?

Mario - Bien, gracias ¿y vos?

Ana - Todo bien, por dicha.

Mario - Bueno, me tengo que ir...me alegro de verte, un día de estos llegáte a mi casa.

a. La oración subrayada es:

$\square$ Un saludo

Una invitación

$\square$ Una despedida

Un mandato

b. ¿Qué le ayuda a entender el significado de la oración subrayada?

$\square$ Algunas palabras

por ejemplo

$\square$ Entiendo todas las palabras

$\square$ No entiendo ninguna palabra

$\square$ La situación

c. ¿Qué dice Ana?

Ana - ¡Diay!, Mario, tanto tiempo sin verte ¿Cómo estás?

Mario - Bien, gracias ¿y vos?

Ana - Todo bien, por dicha.

Mario - Bueno, me tengo que ir...me alegro de verte, un día de estos llegáte a mi casa.

Ana -

2. Segunda situación: Ana se encuentra con su amigo Mario a quien tiene algún tiempo de no ver y dice:

Ana - ¡Diay!, Mario, tanto tiempo sin verte ¿Cómo estás?

Mario - Bien, gracias ¿y vos?

Ana - Todo bien, por dicha.

Mario - Bueno, me tengo que ir...me alegro de verte, un día de estos hagamos algo.

a. La oración subrayada es:

Un saludo

Una invitación

Una despedida

$\square$ Un mandato 
b. ¿Qué le ayuda a entender el significado de la oración subrayada?

$\square$ Algunas palabras

por ejemplo

$\square$ Entiendo todas las palabras

$\square$ No entiendo ninguna palabra

La situación

c. ¿Qué dice Ana?

Ana - ¡Diay!, Mario, tanto tiempo sin verte ¿Cómo estás?

Mario - Bien, gracias ¿y vos?

Ana - Todo bien, por dicha

Mario - Bueno, me tengo que ir...me alegro de verte, un día de estos hagamos algo.

Ana -

\section{Anexo 2}

Pregunta sobre la percepción en cuanto al uso de los enunciados A y B como fórmulas de despedida.

En su opinión, los enunciados "un día de estos llegáte a mi casa" y "un día de estos hacemos algo" qué significado(s) tienen dentro de las situaciones de despedida analizadas.

a. ¿Con que intención se usan?

b. ¿Cuándo se usan? 